\title{
Las Estrías Olfatorias del Ser Humano y Propuesta de Inclusión en la Terminologia Anatomica de Estría Olfatoria Intermedia
}

\author{
Olfactory Stria in the Human Being and Proposal for Inclusion \\ in Terminologia Anatomica of Olfactory Intermediate Stria
}

\author{
Jorge Eduardo Duque Parra**;*; José Fernando Marín Arias ${ }^{* * *}$ \& Félix Jhon César Peláez Cortés*****
}

DUQUE PARRA, J. E.; MARÍN ARIAS, J. F. \& PELÁEZ CORTÉS, F. J. C. Las estrías olfatorias del ser humano y propuesta de inclusión en la Terminologia Anatomica de estría olfatoria intermedia. Int. J. Morphol., 34(4):1411-1413, 2016.

RESUMEN: Las estrías olfatorias del ser humano son dos divisiones del tracto olfatorio, tradicionalmente identificadas como medial y lateral, reconocibles a nivel del trígono olfatorio y asociadas con la corteza piriforme. El objetivo del presente trabajo fue estudiar tomando como referentes treinta y seis nervios olfatorios de cerebros frescos, la presencia de más de dos estrías olfatorias y verificar si hay estría olfatoria intermedia. Se concluye que las estrías olfatorias son variables en número de dos a tres y que se debe incluirse en la Terminologia Anatomica el término estría intermedia.

PALABRAS CLAVE: Estría olfatoria; Estría medial; Estría intermedia; Estría lateral; Nervio olfatorio.

\section{INTRODUCCIÓN}

El sentido de la olfacción es muy importante en los seres humanos, pudiendo jugar un gran papel en la evolución de la dieta, el hábitat y las conductas sociales (Shepherd, 2004), como la búsqueda de alimentos, la localización de predadores, la localización de presas y la detección de individuos (Maier et al., 2011). A pesar del reducido repertorio de genes para esta sensación en los seres humanos, sorprendentemente poseemos un buen sentido del olfato (Laska et al., 2000).

Uno de los elementos involucrados en la olfacción para considerar desde lo estructural, son las estrías olfatorias, divisiones del tracto olfatorio (Dauber, 2007) que transmiten información, establecida de manera tradicional, por dos bandas de fibras ubicadas a nivel del trígono olfatorio (Leonard, 1984; Damodaran et al., 2014) y que en el ser humano son descritas como una lateral y otra medial -esta última nombrada por otros como fascículo olfatorio frontalconstituidas por fibras amielínicas (Mancall \& Brock, 2010).

Dichas estrías olfatorias están consignadas en la Terminologia Anatomica con la referencia A14.1.09.436 (Federative Committee on Anatomical Terminology, 1998).
Para otros, las estrías olfatorias son tres (Sinelnikov, 1986; Delmas, 1981; Leboucq et al., 2013; Mancall \& Brock; Khale \& Frotscher, 2003; Gottfried, 2006): 1. la estría olfatoria lateral, la más abundante en fibras del tracto olfatorio (Allison, 1954; Damodaran et al.) y la más poderosa (Sinelnikov), importante y larga, la cual sigue el margen lateral de la sustancia perforada anterior (Mancall \& Brock) proyectándose al lobo temporal (Leboucq et al.) como fina estructura, en las que se acumulan fibras nerviosas longitudinales, que dividen el tracto olfatorio, formando un arco que sigue el borde más lateral de la sustancia perforada anterior, pasando con su mayoría de fibras a través del limen insular (Mancall \& Brock; Sinelnikov; Damodaran et al.), para llegar al giro parahipocampal (Sinelnikov) y posteriormente al giro periamigdaloide (Dauber) y el cuerpo amigdaloide (Sinelnikov), elementos de la corteza piriforme (Damodaran et al.). 2. La estría olfatoria medial, cuyas fibras continúan hacia la banda diagonal (Mancall \& Brock), terminan en el área subcallosa alrededor del giro paraterminal (Dauber; Sinelnikov), en los núcleos septales (Leboucq et al.) y en parte en el giro fasciolar y en el giro dentado (Sinelnikov). 3. La estría intermedia -o media- (Sinelnikov), que pasa por el centro del trígono, tiene fibras que terminan en la sustancia per-

\footnotetext{
* Departamento de Ciencias Básicas. Programa de Medicina. Universidad de Caldas, Colombia.

** Departamento de Ciencias Básicas Biológicas. Universidad Autónoma de Manizales, Colombia.

*** Departamento de Salud Pública. Programa de Medicina. Universidad de Caldas, Colombia.

***** Departamento de Básico Clínico. Programa de Medicina. Universidad de Caldas, Colombia.
} 
forada anterior, en un pequeño tubérculo olfatorio (Mancall \& Brock), otra parte pasa por la comisura anterior al lado opuesto (Sinelnikov) y los axones de la sustancia perforada se dirigen al fornix y después por la fimbria para llegar al uncus (Sinelnikov); esta última estría es considerada una estructura arcaica (Leboucq et al.). Para otros, el número de estrías olfatorias, puede ser más de tres (Khale \& Frotscher).

\section{MATERIAL Y MÉTODO}

Se valoraron en inspección directa de manera aleatoria, 18 especímenes frescos (36 nervios). Como criterios de inclusión se consideró que no hubieran fallecido por traumas encefálicos o presentaran malformaciones, ni procesos tumorales macroscópicos que afectaran la morfología de la zona a estudiar, y que no fueran fruto de traumatismos o con alteraciones morfológicas, consecuentes con la manipulación posterior a su extracción del cadáver. Se analizó la región del trígono olfatorio y alrededor de la sustancia perforada anterior en el hemisferio derecho y en el izquierdo. Se registró la existencia de las estrías olfatorias en los trígonos correspondientes, documentándose mediante fotografía y cuantificándose la presencia de dichos elementos.

\section{RESULTADOS}

De un total de 18 encéfalos disecados (36 nervios olfatorios) en la región del trígono olfatorio, se encontró que las estrías olfatorias eran evidentes en su recorrido a nivel de la corteza piriforme, con variaciones que iban de 2 a 3 estrías olfatorias, en un hemisferio o en ambos hemisferios. Para el caso de presentación de 2 estrías en el hemisferio derecho (Fig. 1) y 3 en el izquierdo fue 1 (5,55\%) (Fig. 2), 3 estrías en el derecho y 2 en el izquierdo fueron $11(61,11 \%)$, para 2 en el derecho y 2 en el izquierdo fueron $5(27,7 \%)$ y en 1 caso los dos hemisferios presentaron de a 3 estrías respectivamente $(5,55 \%)$.

\section{DISCUSIÓN}

De forma clásica en la Terminologia Anatomica y otros escritos, se indica la existencia de dos estrías olfatorias para el ser humano (Damodaran et al.; Schünke et al., 2010; Schumacher; Leonard; Dauber; Allison; Federative Committee on Anatomical Terminology), más en otros referentes de la literatura, aparecen indicadas por algunos, tres estrías (Sinelnikov; Delmas; Leboucq et al.; Gottfried; Mancall \& Brock), aspecto concordante con lo encontrado en el presente trabajo, donde en 13 trígonos $(72,22 \%)$ había presencia de al menos en un trígono olfatorio con tres estrías olfatorias. Con ello se demuestra que las estrías olfatorias en proporción, son más abundantes en la presentación de tres, pero en ningún caso encontramos más de tres, por lo que no hay coincidencias con otros autores (Khale \& Frotscher), aspecto que lleva a considerar el que

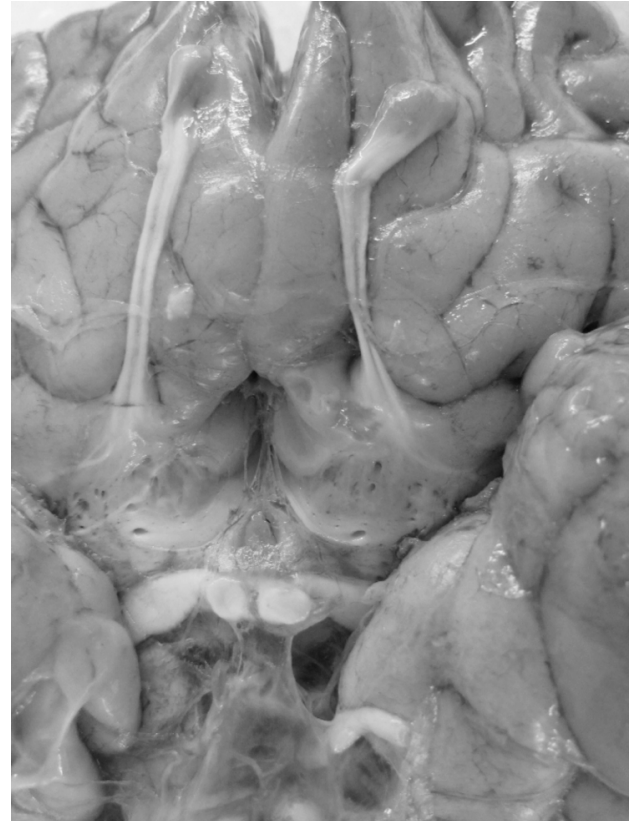

Fig. 1. Se identifican en ambos nervios olfatorios dos estrías olfatorias.

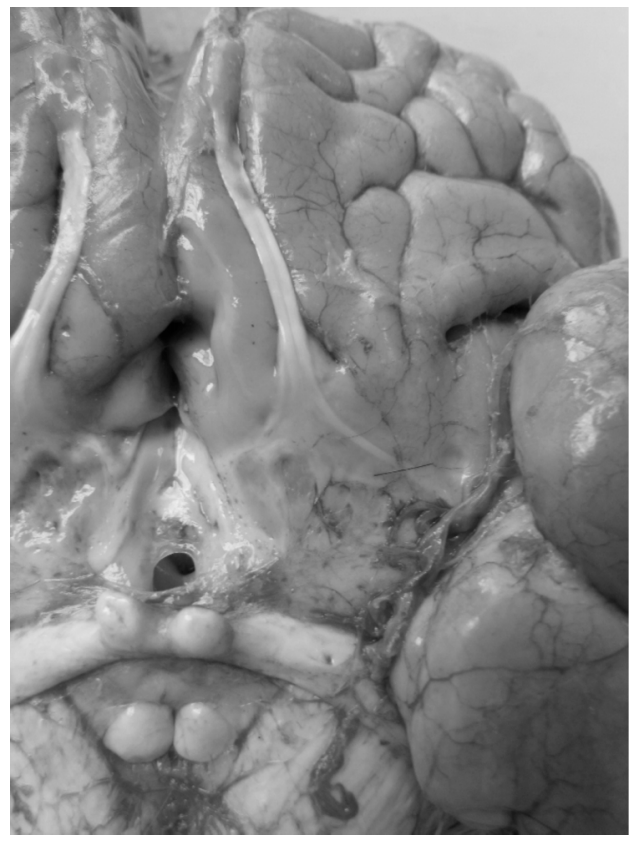

Fig. 2. Se identifican en el hemisferio izquierdo tres estrías olfatorias.

deba incluirse en la Terminologia Anatomica la estría olfatoria intermedia, más como una estructura constante que como variación. Aunque se ha citado que solo la estría olfatoria lateral es la única significativa en el ser humano (Patel \& Pinto, 2014), estos aspectos neuroanatómicos de las estrías olfatorias, pueden revelar dominancia hemisférica para la olfacción en la mayoría de los casos, o al me- 
nos mayor diversidad de proyecciones a diversos sectores encefálicos, por lo que el conocimiento neuroanatómico de esta diversidad, implicaría proyecciones de estas fibras en cantidad y conexiones a diversas áreas funcionales del cerebro, y cuyo papel implica conexiones que podrían redundar en la conducta, la memoria, las emociones, las sensaciones placenteras (Damodaran et al.; Pinto, 2011), la seguridad, la nutrición, la sexualidad y el estado de ánimo (Pinto), las sensaciones displacenteras, además de participar como elementos aferentes de la corteza rinal, en la evocación de vivencias pasadas y efectos fisiológicos como náuseas y emesis. En conclusión, el número de estrías olfatorias en el cerebro humano es variable y su número puede ser de 2 a 3 en cada hemisferio, con cuantificación que va desde 2 y 3 bilateralmente o 2 y 3 , tanto en lado derecho como izquierdo. Pueden ser dos en un hemisferio y tres en otro en un mismo individuo, lo que demuestra la enorme variabilidad de presentación, aunque con predominio de presentar al menos tres estrías olfatorias. La estría olfatoria intermedia es una estructura que debe incluirse en la Terminologia Anatomica.

DUQUE PARRA, J. E.; MARÍN ARIAS, J. F. \& PELÁEZ CORTÉS, F. J. C. Olfactory stria in the human being and proposal for inclusion in Terminologia Anatomica of olfactory intermediate stria. Int. J. Morphol., 34(4):1411-1413, 2016.

SUMMARY: The human olfactory stria are two divisions of the olfactory tract, traditionally identified as medial and lateral olfactory stria, recognizable at the level of the olfactory trigone, and associated with the piriform cortex. The aim of this work was to study taking as reference thirty six fresh brain's olfactory nerves, the presence of more than two olfactory stria and check for intermediate olfactory stria. It is concluded that the olfactory stria are variable in number from two to three and this should be included in the Terminologia Anatomica as the term stria intermedia.

KEY WORDS: Intermedia stria; Lateral stria; Medial stria; Olfactory nerve, Olfactory stria.

\section{REFERENCIAS}

Allison, A. C. The secondary olfactory areas in the human brain. $J$. Anat., 88(4):481-8, 1954.

Dauber, W. Pocket Atlas of Human Anatomy. Stuttgart, Thieme, 2007.

Damodaran, O.; Rizk, E.; Rodriguez, J. \& Lee, G. Cranial nerve assessment: a concise guide to clinical examination. Clin. Anat., 27(1):25-30, 2014.

Delmas, A. Les Voies Olfactives et le Lobe Olfactif: Paleocortex. In: Delmas, A. (Ed.). Voies et Centres Nerveux. 10a ed. Paris, Masson, 1981. pp.218-20.
Federative Committe on Anatomical Terminology (FCAT). Terminologia Anatomica: International Anatomical Terminology. Stuttgart, Thieme, 1998.

Gottfried, J. A. Smell: central nervous processing. $A d v$. Otorhinolaryngol., 63:44-69, 2006.

Khale, W. \& Frotscher, M. Nervous System Sensory Organs. New York, Thieme, 2003.

Laska, M.; Seibt, A. \& Weber, A. 'Microsmatic' primates revisited: olfactory sensitivity in the squirrel monkey. Chem. Senses, 25(1):47-53, 2000

Leboucq, N.; Menjot de Champfleur, N.; Menjot de Champfleur, S. \& Bonafé, A. The olfactory system. Diagn. Interv. Imaging, 94(10):985-91, 2013.

Leonard, C. H. Gray's Pocket Anatomy. The Dissecting Room Companion. New York, Bounty Books, 1984.

Maier, E.; Nord, H.; von Hofsten, J. \& Gunhaga, L. A balance of BMP and notch activity regulates neurogenesis and olfactory nerve formation. PloS One, 6(2):e17379, 2011.

Mancall, E. L. \& Brock, D. G. Gray's Clinical Neuroanatomy. The Anatomic Basis for Clinical Neuroscience. Philadelphia, Saunders, 2010.

Patel, R. M. \& Pinto, J. M. Olfaction: anatomy, physiology, and disease. Clin. Anat., 27(1):54-60, 2014.

Pinto, J. M. Olfaction. Proc. Am. Thorac. Soc., 8(1):46-52, 2011.

Schünke, M.; Schulte, E.; Schumacher, U.; Voll, M. \& Wesker, K. Prometheus. Texto y Atlas de Anatomía. Tomo 3. Buenos Aires, Editorial Médica Panamericana, 2010.

Shepherd, G. M. Unsolved Mystery. The human sense of smell: are we better than we think? PloS Biol., 2(5):E146, 2004.

Sinelnikov, R. D. Atlas de Anatomía Humana. Tomo III. Moscú, Editorial MIR, 1986.

Correspondence to: Jorge Eduardo Duque Parra

Departamento de Ciencias Básicas

Programa de Medicina

Universidad de Caldas

Manizales

COLOMBIA

Email: jorge.duque_p@ucaldas.edu.co

Received: 04-07-2016

Accepted: 17-10-2106 